\title{
Gold nanoparticles attenuate albuminuria by inhibiting podocyte injury in a rat model of diabetic nephropathy
}

\author{
Ghada Alomari $^{1,2}$ - Bahaa Al-Trad ${ }^{2}$. Salehhuddin Hamdan ${ }^{1}$ - Alaa Aljabali ${ }^{3}$. Mazhar Al-Zoubi ${ }^{4}$ Nesreen Bataineh ${ }^{4}$. \\ Janti Qar ${ }^{2} \cdot$ Murtaza M. Tambuwala ${ }^{5}$
}

Published online: 21 October 2019

(C) The Author(s) 2019

\begin{abstract}
Several recent studies have reported that gold nanoparticles (AuNPs) attenuate hyperglycemia in diabetic animal models without any observed side effects. The present study was intended to provide insight into the effects of 50$\mathrm{nm}$ AuNPs on diabetic kidney disease. Adult male rats were divided into three groups ( $n=7 /$ group): control (nondiabetic, ND), diabetic (D), and diabetic treated intraperitoneally with 50-nm AuNPs (AuNPs + D; $2.5 \mathrm{mg} / \mathrm{kg} / \mathrm{day}$ ) for 7 weeks. Diabetes was induced by a single-dose injection of $55 \mathrm{mg} / \mathrm{kg}$ streptozotocin. The result showed that AuNP treatment prevented diabetes-associated increases in the blood glucose level. Reduction in 24-h urinary albumin excretion rate, glomerular basement membrane thickness, foot process width, and renal oxidative stress markers was also demonstrated in the AuNP-treated group. In addition, the results showed downregulation effect of AuNPs in renal mRNA or protein expression of transforming growth factor $\beta 1$ (TGF- $\beta_{1}$ ), fibronectin, collagen IV, tumor necrosis factor- $\alpha$ (TNF- $\alpha$ ), and vascular endothelial growth factor-A (VEGF-A). Moreover, the protein expression of nephrin and podocin, podocyte markers, in glomeruli was increased in the AuNPs + D group compared with the D group. These results provide evidence that 50-nm AuNPs can ameliorate renal damage in experimental models of diabetic nephropathy through improving the renal function and downregulating extracellular matrix protein accumulation, along with inhibiting renal oxidative stress and amelioration of podocyte injury.
\end{abstract}

Keywords Gold nanoparticles $\cdot$ Diabetic nephropathy $\cdot$ Streptozotocin $\cdot$ Rats $\cdot$ Nanomedicine

Ghada Alomari

alomarighada@yahoo.com

$\triangle$ Bahaa Al-Trad

bahaa.tr@yu.edu.jo

$\triangle$ Murtaza M. Tambuwala

m.tambuwala@ulster.ac.uk

1 Department of Bioscience, Faculty of Science, Universiti Teknologi Malaysia, 81310 Skudai, Johor, Malaysia

2 Department of Biological Sciences, Yarmouk University, Irbid 21163, Jordan

3 Faculty of Pharmacy, Department of Pharmaceutical Sciences, Yarmouk University, Irbid, Jordan

4 Faculty of Medicine, Department of Basic Medical Sciences, Yarmouk University, Irbid, Jordan

5 School of Pharmacy and Pharmaceutical Science, SAAD Centre for Pharmacy and Diabetes, Ulster University, Coleraine, County Londonderry, Northern Ireland, UK

\section{Introduction}

Diabetic nephropathy (DN) is the major cause of endstage renal failure and mortality in diabetic patients all over the world [1]. In DN, the first detected histological alteration usually appears on glomeruli as a thickening of the glomerular basement membrane (GBM) and tubular basement membrane (TBM) [2]. Both GBM thickening and glomerular hyperfiltration lead to the progression of microalbuminuria, while the leading cause for renal insufficiency in DN is the mesangium expansion, which develops at a later stage [3]. The expanded mesangium cells restrict and distort glomerular capillaries leading to the collapsing of glomerular capillaries and in turn reducing the filtration surface and decrease creatinine clearance $[2,4]$.

Mesangial expansion and GBM thickening occur as a result of increased expression and accumulation of the extracellular matrix (ECM) proteins such as collagen, 
fibronectin, and laminin in the mesangium and renal tubulointerstitium of the glomerulus and basement membranes [5]. This subsequently resulted from their increased production, decreased degradation, or both which eventually resulting in renal fibrosis and DN [2, 4-6]. Chronic hyperglycemia is the major initiator of these changes through increased oxidative stress, overproduction and action of advanced glycation end products (AGE), and activation of several growth factors, such as transforming growth factor- $\beta 1$ (TGF- $\left.\beta_{1}\right)$ and vascular endothelial growth factor (VEGF-A) [7].

Podocytes are highly specialized cells that act as size and charge barriers to protein and maintain the integrity of the glomerular filtration barrier [8]. One of the significant events in the pathogenesis of $\mathrm{DN}$ is the progressive podocyte injury, which expresses the recession of the podocyte foot processes that lead to defacement, loss of slit diaphragm proteins, detachment, and finally cell apoptosis [9]. Different studies have shown that the development of albuminuria and the acceleration of glomerular structural abnormalities in DN are associated with podocyte injury and loss [8].

Despite the available modern therapies that can delay the onset of DN (glucose and blood pressure control drugs), numerous patients continue to show progressive renal damage. Thus, it is extremely necessary to identify novel therapeutic strategies that could specifically target the progression of DN [10]. Recently, nanoparticles (NPs) have been widely used in biomedical applications because they can pass through biological barriers and their ability to enhance the bioavailability of therapeutic agents [11, 12]. Several studies have revealed that gold nanoparticles (AuNPs) exhibited anti-hyperglycemic, anti-inflammatory, and antioxidative activities in streptozotocin (STZ)-induced diabetic animal model $[13,14]$. Hyperglycemia and oxidative stress are important factors in the development of diabetic microvascular complications including DN $[15,16]$. Recent studies have demonstrated that pomegranate peel extract-stabilized 20-nm AuNPs and 13nm naked AuNPs reduced STZ-induced renal fibrosis, inflammation, and oxidative stress [17, 18]. However, the maximized kidney targeting at the cellular (mesangium), tissue, and organ levels will be achieved via the usage of 50-nm AuNPs [19]. To the best of our knowledge, the effects of 50-nm AuNPs on the albuminuria, podocyte injury, oxidative stress, inflammation, and angiogenesis have not yet been studied in DN. The goals of the current study were to demonstrate the effects of $50-\mathrm{nm}$ AuNPs in the STZinduced diabetic rats and to explore their protective effects against diabetic albuminuria and podocyte injury.

\section{Materials and methods}

\section{Materials}

Sodium citrate trihydrate, $\mathrm{HAuCl}_{4}$, RNAlater solution, and Periodic Acid Schiff staining kit were all provided from Sigma-Aldrich, (St. Louis, USA). Streptozotocin and antibodies against TGF- $\beta_{1}$ (Cat. No. sc-146) were both purchased from Santa Cruz biotechnology (Santa Cruz, USA). Albumin Rat ELISA kit, HRP/DAB IHC Detection KitMicropolymer, and the primary antibodies against Collagen IV (Cat. No. ab6586), Fibronectin (Cat. No.ab2413), nephrin (Cat. No.ab1830990), podocin (Cat.No.ab50339) were all provided from Abcam (Cambridge, UK). The superoxide dismutase assay kit was purchased from Cayman Chemical, (Ann Arbor, USA). Catalase assay kit was provided from Abbexa (Cambridge, UK). The Total RNA extraction kit was from iNtRON Biotechnology, Inc. (Seongnam, Korea). cDNA reverse transcription kit and SYBR green PCR master mix were purchased from Takara (Beijing, China). All primers were synthesized by Integrated DNA Technologies, INC. (Coralville, IA, USA). All other chemicals, buffers, and solvents used were of pure analytical grade.

\section{Synthesizing and characterization of AuNPs}

The preparation and characterization of AuNPs were mentioned in our previous report [20]. In brief, $300 \mu \mathrm{l}$ of an aqueous solution of $1 \%(\mathrm{w} / \mathrm{v}) \mathrm{HAuCl}_{4}$ was added to $30 \mathrm{ml}$ of ultrapure $\mathrm{H}_{2} \mathrm{O}$ and brought to boil on magnetic hotplate (BT lab system, UK) while gently stirring at $150 \mathrm{rpm}$. Once the solution reaches boiling, $900 \mu \mathrm{l}$ of freshly prepared aqueous of $1 \%$ $(\mathrm{w} / \mathrm{v})$ sodium citrate trihydrate was added to the reaction mixture. The reaction was allowed to proceed for $10 \mathrm{~min}$ where the AuNP formation was completed as indicated by the color transformation to ruby-red from yellow. Size and morphology of the resulted AuNPs were characterized using a scanning electron microscope (Quanta FEG SEM, Thermo Fisher Scientific, UK), dynamic light scattering (DynaPro Titan Wyatt Technology Corporation) equipped with a laser wavelength of $\lambda=830 \mathrm{~nm}$, scattering angle of $2^{\circ}$ equipped with Dynamics software version 6.9.2.11, and zeta potential instruments were recorded on (Malvern Instruments Zetasizer-Nano ZS) in which $0.2 \mathrm{mg}$ was suspended in $1 \mathrm{ml}$ of $0.1 \mathrm{M}$ sodium phosphate buffer $\mathrm{pH}$ 7.0. Zeta cells were pre-equilibrated at $21{ }^{\circ} \mathrm{C}$ with the buffer, and data were presented from 3 independent synthesis.

\section{Induction of diabetes and experimental protocols}

All animal experimental procedures were carried out in accordance with the National Institutes of Health guidelines for the care and use of laboratory animals and approved by the 
committee of animal ethics at Yarmouk University (permission number: YU-20/12/2017). Adult male Wistar rats weighting 250 to $300 \mathrm{~g}$ were maintained under specific pathogen-free conditions at the animal house unit at Yarmouk University. After 1 week of adaptation, diabetes was induced by a single intraperitoneal injection of streptozotocin (STZ) $(55 \mathrm{mg} / \mathrm{kg}$; following overnight fasting). Three days after STZ injection, rats with a blood glucose level over $250 \mathrm{mg} / \mathrm{dl}$ were considered diabetic. Rats were divided into three groups ( $n=7 /$ group): control (non-diabetic, $\mathrm{ND}$ ), diabetic (D), and diabetic treated intraperitoneally with $50 \mathrm{~nm}$ AuNPs (AuNPs + D; $2.5 \mathrm{mg} / \mathrm{kg}$ ) daily for 7 weeks.

The size, dose, and the route of administration of AuNPs used in the present study were selected based on the previous study that reported that intraperitoneal administration of spherical, 50-nm AuNPs showed anti-hyperglycemia and anti-oxidative effect in STZ-induced diabetic mice without toxic effect over the vital organs [13].

\section{Urine, blood, and tissue collection}

At the end of 7 weeks of treatment, the rats were placed in metabolic cages 1 day before sacrifice, and urine was collected for $24 \mathrm{~h}$ for the analysis of urine albumin concentration and the urine output. Then, the animals were weighed and lightly anesthetized with ethyl ether, and blood samples were collected (via cardiac puncture). The animals were sacrificed using an overdose of ethyl ether. The right kidney was removed and then transferred into RNAlater solution for the real-time PCR analysis. The left kidney was fixed with $4 \%$ paraformaldehyde for morphological and immunohistochemical analysis.

\section{Measurements of blood glucose, 24-h urinary albumin excretion rate, and renal oxidative stress markers}

The blood glucose level was determined by glucometer (Accu-Chek Performa, Roche Diagnostics). Urine samples were centrifuged at $4{ }^{\circ} \mathrm{C}$ and $2000 \mathrm{rpm}$ for $10 \mathrm{~min}$. The urinary albumin in the supernatant was measured using Albumin Rat ELISA kit according to the manufacturer's protocols.

Kidney tissues were homogenized in protein extraction buffer containing PBS and protease inhibitor. Commercially available kits were used according to the manufacturer protocols to identify the activities of superoxide dismutase and catalase. The renal malondialdehyde (MDA) level, a marker of lipid peroxidation, was assessed by a method described previously [21]. In brief, renal tissue homogenate $(0.2 \mathrm{mg}$ protein $/ \mathrm{ml}$ ) was combined with $2.0 \mathrm{ml}$ of thiobarbituric acid (TBA), trichloroacetic acid (TCA), and TCA-TBA-HCI and was mixed thoroughly. Then, the solution was heated for $15 \mathrm{~min}$ in a boiling water bath, and after cooling, the flocculent precipitate was removed by centrifugation at $1000 \mathrm{~g}$ for
$10 \mathrm{~min}$. The absorbance of the sample was determined at $535 \mathrm{~nm}$ against a blank that contains all the reagents minus the lipid.

\section{Morphology studies}

After the fixation in $4 \%$ paraformaldehyde, the tissues were dehydrated in ascending series of alcohol, cleared in xylene, embedded in paraffin, sectioned at $4 \mu \mathrm{m}$, and stained with hematoxylin and eosin (H\&E) and periodic acid-Schiff. For electron microscopy study, renal cortical tissues were cut into $1-\mathrm{mm}^{3}$ cubes, fixed in $2.5 \%$ glutaraldehyde dissolved in $0.1 \mathrm{M}$ sodium cacodylate buffer $(\mathrm{v} / \mathrm{v} \%)(\mathrm{pH} 7.4)$ for $1 \mathrm{~h}$, and then washed in the same buffer. The tissue fragments were post-fixed in sodium cacodylate-buffered $1 \% \mathrm{OsO}_{4}(\mathrm{v} / \mathrm{v} \%)$ for $2 \mathrm{~h}$, dehydrated, and embedded in Spurr's resin. Ultra-thin sections were cut using ultra-microtome (Reichert-Jung, Austria), contrasted with $4 \%$ uranyl acetate for $45 \mathrm{~min}$, and subsequently incubated with lead citrate for $4 \mathrm{~min}$ at room temperature. Sections were examined under a Hitachi electron microscope (HT7700-Tokyo). The GBM thickness was measured and calculated as previously detailed [22]. The degree of podocyte detachment was calculated as the mean of foot process width (FPW). From each photograph, the arithmetic mean of the FPW was calculated according to the equation below [23]:

$\mathrm{FPW}=\frac{\pi}{4} \times \frac{\sum \mathrm{GBM} \text { length }}{\sum \text { foot process }}$

where GBM length is the total GBM length measured in each photo and foot process is the total number of foot processes counted in that photo. To correct for supposed random difference in the angle of section relative to the long axis of the podocyte, the correction factor of $\pi / 4$ was used. GBM length was calculated by computerized measurement using the ImageJ software. Podocyte foot processes number was computed by hand [23].

\section{Immunohistochemistry}

Immunohistochemical staining was performed with mouse and rabbit-specific HRP/DAB IHC Detection Kit-Micropolymer, according to the manufacturer's protocol using specific primary antibodies against TGF- $\beta_{1}$, ECM proteins, and podocyte markers as follows: collagen IV (1:100), fibronectin (1:50), nephrin (1:100), podocin (1:50), and TGF- $\beta_{1}(1: 100)$. Negative controls were run by replacing the primary antibody with PBS. Sections were examined under an Optika microscope and evaluated using Fiji ImageJ software. 


\section{Real-time PCR}

Total RNA was extracted from RNAlater-preserved kidney tissues using Total RNA extraction kit according to the manufacturer's protocols. RNA quantity was determined using QuantiFluor RNA System (Promega, Madison, USA) and Quantus Fluorometer (Promega, Madison, USA). A cDNA reverse transcription kit was used to prepare the cDNA template, according to the manufacturer's instructions. Quantitative real-time PCR was conducted using LineGene 9600 Real-Time PCR system (Bioer Technology Co., Binjiang, China), with the SYBR green PCR master mix, at $95^{\circ} \mathrm{C}$ for $2 \mathrm{~min}$ and 45 cycles of $95^{\circ} \mathrm{C}$ for $30 \mathrm{~s}$, and $60^{\circ} \mathrm{C}$ for $30 \mathrm{~s}$. GAPDH was used as a non-regulated reference gene. PCR primers of TGF- $\beta_{1}$, VEGF-A, collagen IV, fibronectin, and TNF- $\alpha$ primers were designed using Primer3 (http:// www.ncbi.nlm.nih.gov/tools/primer-blast/) software and synthesized by IDT (Table 1). To ensure the specificity of the primers, a gel was run, in which a single band of expected size was obtained. The fold changes in the mRNA expression were determined using the $2^{-\Delta \Delta \mathrm{CT}}$ method [24].

\section{Statistical analysis}

All data are expressed as the mean $\pm \mathrm{SEM}$; the minimum number of replicates used in the analysis was 6 . Differences between groups were calculated by one-way analysis of variance (ANOVA) using SPSS software (SPSS Inc., Chicago, IL). The significant value of difference was considered when the $P$ value $<0.05$.

\section{Results}

\section{Characterization of AuNPs}

Characterization of the generated particles revealed that the generated AuNPs mainly consist of spherical particles with an average hydrodynamic diameter of $51.8 \pm$
$0.7 \mathrm{~nm}$ and with a polydispersity index of $0.26 \%$. Furthermore, the surface zeta potential related to the surface charge was $-40.0 \pm 0.2$ [20].

\section{Effect of AuNPs on metabolic and biochemical parameters}

After 7 weeks of STZ administration, diabetic animals showed significant increases in the blood glucose level, kidney weight/body weight ratio, and 24-h urinary albumin excretion rate, indicating that we successfully established an experimental animal model of type 1 diabetes possessing DN $(P<0.05$; Table 2). Treatment with AuNPs did not affect the kidney weight/body weight ratio in diabetic rats significantly. However, the AuNP treatment significantly decreased blood glucose level and 24-h urinary albumin excretion rate compared with the D group $(P<0.05$; Table 2).

\section{Renal histopathological and ultrastructural changes}

The histological morphology showed organized intact glomeruli, Bowman's capsule, and tubular structure in the ND group (Fig. 1a). In contrast, the histological examination of the renal tissue in the D group showed disorganized architecture characterized by expansion in urinary space and closure of capillary loops and tubular atrophy and increased vacuolization of renal tubular epithelial cells. However, AuNP treatment ameliorates these abnormal histological findings in the AuNPs + D group. Light microscopy sections of PAS staining showed mesangial expansion and thick basement membranes in the $\mathrm{D}$ group compared with the ND group) Fig. 1b). While in AuNPs + D group, GBM showed normal thickness with decreased in mesangial matrix deposition.

Several ultrastructural abnormalities were present in the electron microscopy sections (Fig. 2) in the D group such as diffused thickening of the GBM, a reduction in the podocyte number, and diffused foot process effacement, along with a reduced podocyte per glomerulus ratio. Treatment of diabetic rats with AuNPs ameliorated the above pathogenic findings.

Table 1 Sequences of primers used for quantitative real-time PCR

\begin{tabular}{lll}
\hline Gene $^{1}$ & Forward (5-3) & Reverse (5-3) \\
\hline GAPDH & ATG GTG AAG GTC GGT GTG & GAA CTT GCC GTG GGT AGA \\
TGF- $\beta_{1}$ & GTG GAG CAA CAC GTA GAA C & TCC TTG GTT CAG CCA CT \\
VEGF-A & CGA ACA GAG AGA GGG ACA GG & GTC TGT CTG TCT GTC CGT CA \\
Fibronectin & TGA CCG ACG CTA CAG AAA CT & TTG AGC GTG TAC AGG TGG AT \\
TNF- $\alpha$ & TTC GGA ACT CAC TGG ATC CC & GGA ACA GTC TGG GAA GCT CT \\
Collagen IV & TTG GCT TTC CTG GTA GTC GT & CAA CCT TTC CTG CTT CAC CC \\
\hline
\end{tabular}

${ }^{1} G A P D H$, glyceraldehyde 3-phosphate dehydrogenase; $T G F-\beta_{1}$, transforming growth factor- $\beta 1$; VEGF-A, vascular endothelial growth factor-A; $T N F$ $\alpha$, tumor necrosis factor- $\alpha$ 
Table 2 Effect of AuNPs on metabolic and biochemical parameters

\begin{tabular}{|c|c|c|c|c|c|}
\hline \multirow[t]{2}{*}{ Parameters } & \multirow[t]{2}{*}{ ND } & \multicolumn{2}{|l|}{$\mathrm{D}$} & \multicolumn{2}{|l|}{ AuNPs + D } \\
\hline & & Mean \pm SEM & $P$ value* & Mean \pm SEM & $P$ value $\$$ \\
\hline Blood glucose (mg/dl) & $113.1 \pm 5.6$ & $340.83 \pm 42.11 *$ & $<0.0001$ & $144.1 \pm 25.48^{\$}$ & $<0.0001$ \\
\hline Kidney weight/body weight ratio & $0.60 \pm 0.02$ & $1.12 \pm 0.02 *$ & $<0.0001$ & $1.035 \pm 0.085$ & 0.1992 \\
\hline Urinary albumin excretion rate ( $\mathrm{mg} /$ day) & $0.14 \pm 0.04$ & $0.68 \pm 0.06^{*}$ & $<0.0001$ & $0.32 \pm 0.07^{\$}$ & 0.0004 \\
\hline
\end{tabular}

Data represent the mean \pm SEM. $* P$ value compared with the ND group. ${ }^{\$} P$ value compared with D. $N D$, non-diabetic; $D$, diabetic; $A u N P s$, gold nanoparticles

\section{Effect of AuNPs on renal oxidative stress markers}

Diabetic animals showed significant decreases in the renal tissue SOD and catalase activities and increase in the MDA level compared with the ND group $(P<0.05$; Table 3$)$. Notably, AuNP administration restored the renal SOD and catalase activity and the MDA level to the normal values.

\section{Effect of AuNPs on the renal expression of fibrosis, angiogenesis, and inflammation markers}

The immunohistochemistry analysis demonstrated that diabetes was associated with an increase in the intensity of immunostaining for collagen IV, fibronectin, and TGF- $\beta_{1}$ (Fig. 3a, b), which are the major ECM proteins that lead to the development of the mesangial matrix expansion in the status of DN [25]. Consistent with the immunohistochemical findings, the levels of mRNA encoding for fibronectin and TGF- $\beta_{1}$ were significantly greater in the $\mathrm{D}$ than in the ND group (Fig. $4 \mathrm{a}, \mathrm{b} ; P<0.05$ ). However, the collagen IV mRNA levels did not differ significantly between D and AuNPs $+\mathrm{D}$ groups (Fig. 4c; $P>0.05$ ). The treatment of diabetic rats with AuNPs downregulates the expression of these fibrosis markers, suggesting that AuNP treatment inhibits the progression of renal fibrosis in the AuNPs + D group.

Upregulation in the expression of VEGF-A, an angiogenic factor, and TNF- $\alpha$, an inflammatory marker is often observed in the DN. Figure $4 \mathrm{~d}$ and e show that the mRNA expression levels of VEGF-A and TNF- $\alpha$ were significantly increased in the D group compared with the ND group $(P<0.05)$. However, the treatment with AuNPs downregulates the expression of VEGF-A and TNF- $\alpha$ in the AuNPs + D group.

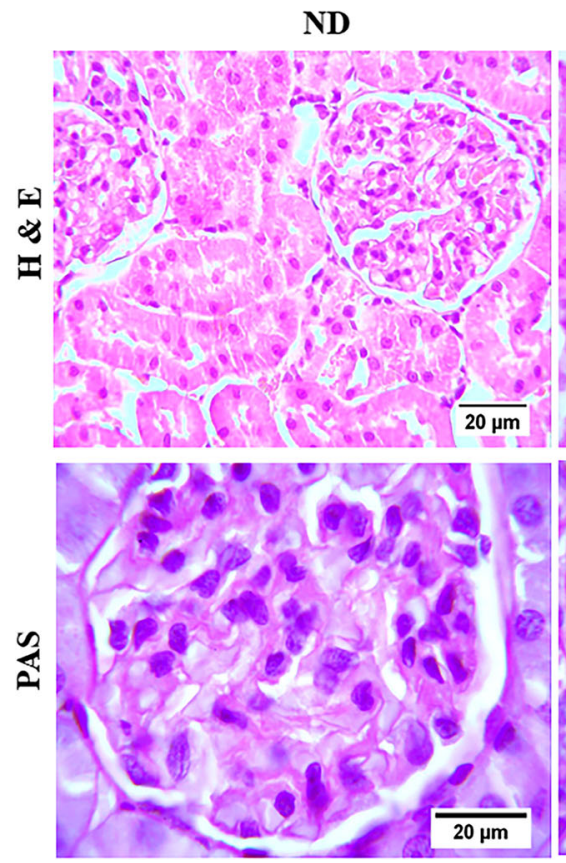

Fig. 1 a H\&E-stained sections (magnification, $\times 400$ ) show intact and organized structure glomerulus in the ND group. Disorganized architecture of glomerulus in the D group and normal glomeruli the AuNPs + D group. Asterisk indicates expansion in urinary space. b PAS-stained sections (magnification, $\times 1000$ ) show that the percentage of the PAS-

D
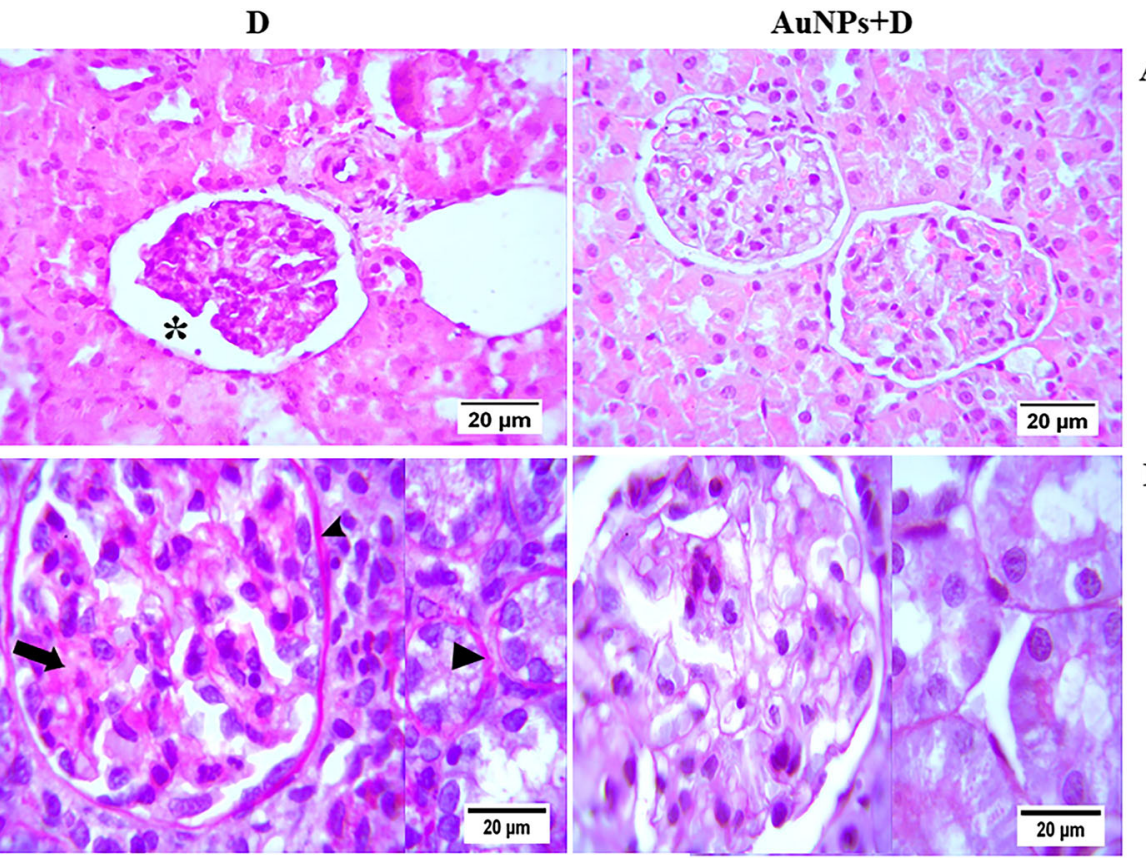

positive material, indicative of increased fibrosis, was significantly increased in the D group compared with the ND group. Arrow head: basement membrane thickening, arrow: mesangial expansion. AuNP treatment inhibited the increase in the PAS-positive material in the AuNPs + D group 


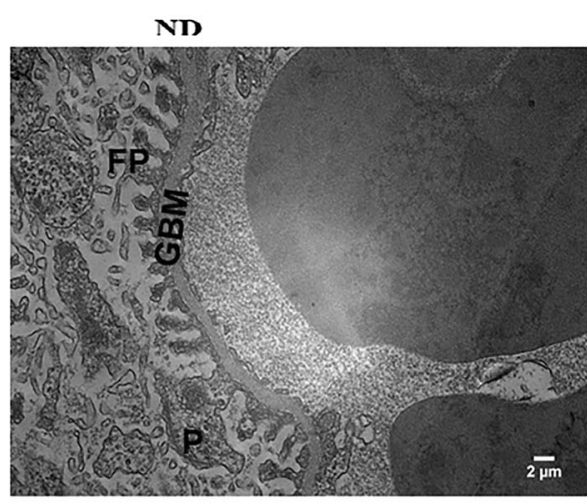

\section{D}
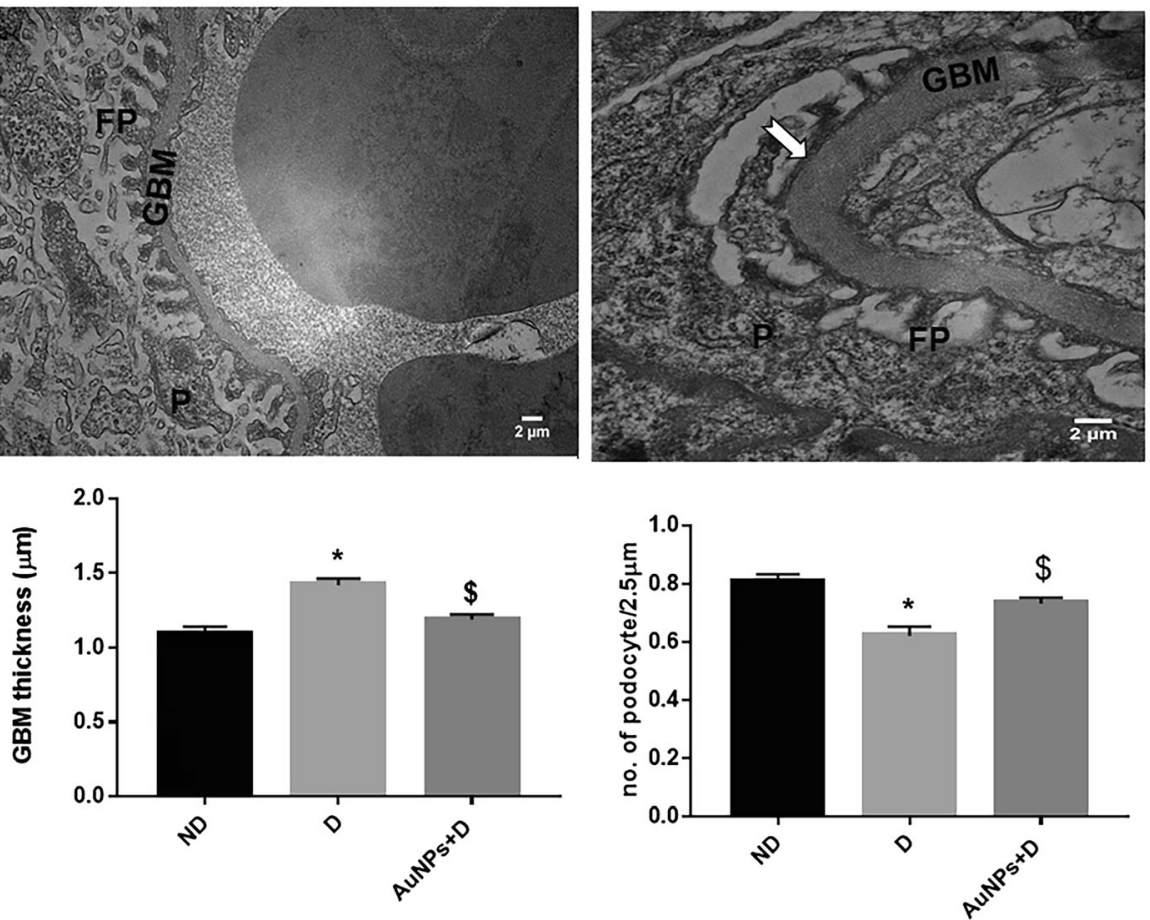

AuNl's+D
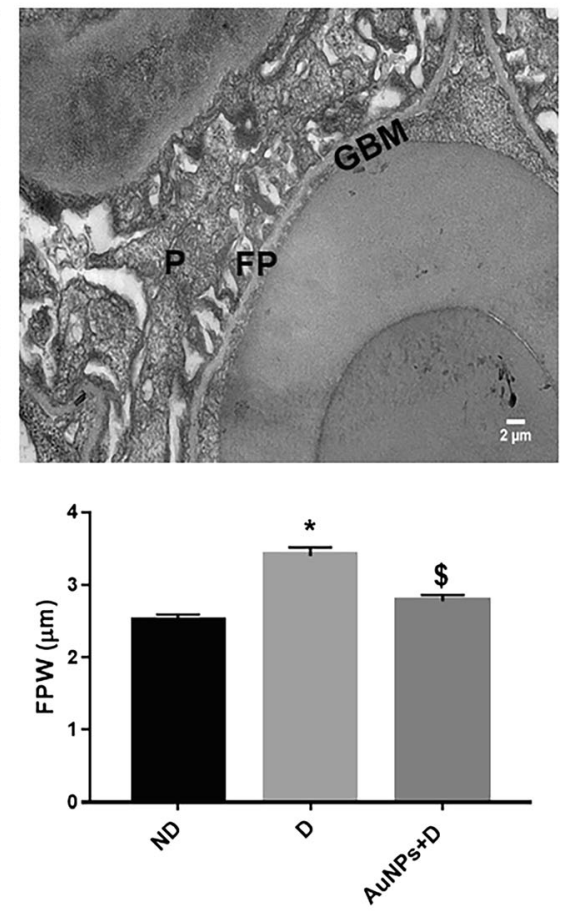

Fig. 2 Glomerular ultrastructure in the different treated groups. a Representative electron microscopy images of podocyte foot processes and the GBM (magnification: ND and AuNPs $+\mathrm{D} \times 6000, \mathrm{D} \times 8000$ ). White arrow: foot process effacement. b Glomeruli were evaluated to quantify the GBM thickness. $* P<0.05$ compared with the ND group. ${ }^{\$} P<0.0001$ compared with $\mathrm{D}$, number of podocyte. $* P<0.05$ compared

\section{Effects of AuNP treatment on the expression of podocyte markers (nephrin and podocin)}

The expression intensity and the distribution pattern of nephrin and podocin in the glomeruli were assessed by immunohistochemical staining (Fig. 5a). In the D group, the expression intensity of nephrin and podocin were significantly decreased compared with the ND group (Fig. 5b, $P<0.05$ ). However, AuNPs fully improved the immunohistochemical staining intensity of both podocyte markers in the AuNPs + D group. with the ND group. ${ }^{\$} P<0.0025$ compared with $\mathrm{D}$ and FPW. $* P<0.05$ compared with the ND group. ${ }^{\$} P<0.0001$ compared with D. Data represent the mean \pm SEM. ND, non-diabetic; D, diabetic; AuNPs, gold nanoparticles; GBM, glomerular basement membrane thickness; FPW, foot process width; $P$, podocyte

\section{Discussion}

Due to their unique physical and chemical properties, many pieces of research have been conducted to elucidate their potential efficiency in the treatments of many diseases. Accordingly, using AuNPs as a promising antidiabetic agent just started to emerge [13, 26-28]. To explore the effect of AuNPs on the DN, an experimental DN similar to DN in type 1 diabetic patient was established successfully in this study that characterized by hyperglycemia and albuminuria that were associated with the loss of podocytes, effacement of foot

Table 3 Effect of AuNPs on renal oxidative stress markers

\begin{tabular}{lllll}
\hline Parameters & ND & D & & AuNPs + D \\
\cline { 3 - 5 } & & Mean \pm SEM & $P$ value* & Mean \pm SEM \\
\hline SOD $(\mathrm{U} / \mathrm{mg})$ & $2.84 \pm 0.14$ & $2.07 \pm 0.36^{*}$ & 0.043 & $2.90 \pm 0.20^{\$}$ \\
Catalase $(\mathrm{U} / \mathrm{mg})$ & $5.54 \pm 0.89$ & $1.67 \pm 0.31^{*}$ & 0.015 & $4.82 \pm 1.29^{\$}$ \\
MDA $(\mu \mathrm{mol} / \mathrm{mg})$ & $0.12 \pm 0.04$ & $0.49 \pm 0.17^{*}$ & 0.014 & 0.046 \\
\hline
\end{tabular}

Data represent the mean \pm SEM. ${ }^{*} P$ value compared with the ND group. ${ }^{\$} P$ value compared with D. $N D$, non-diabetic; $D$, diabetic; $A u N P s$, gold nanoparticles; $S O D$, superoxide dismutase; $M D A$, malondialdehyde 


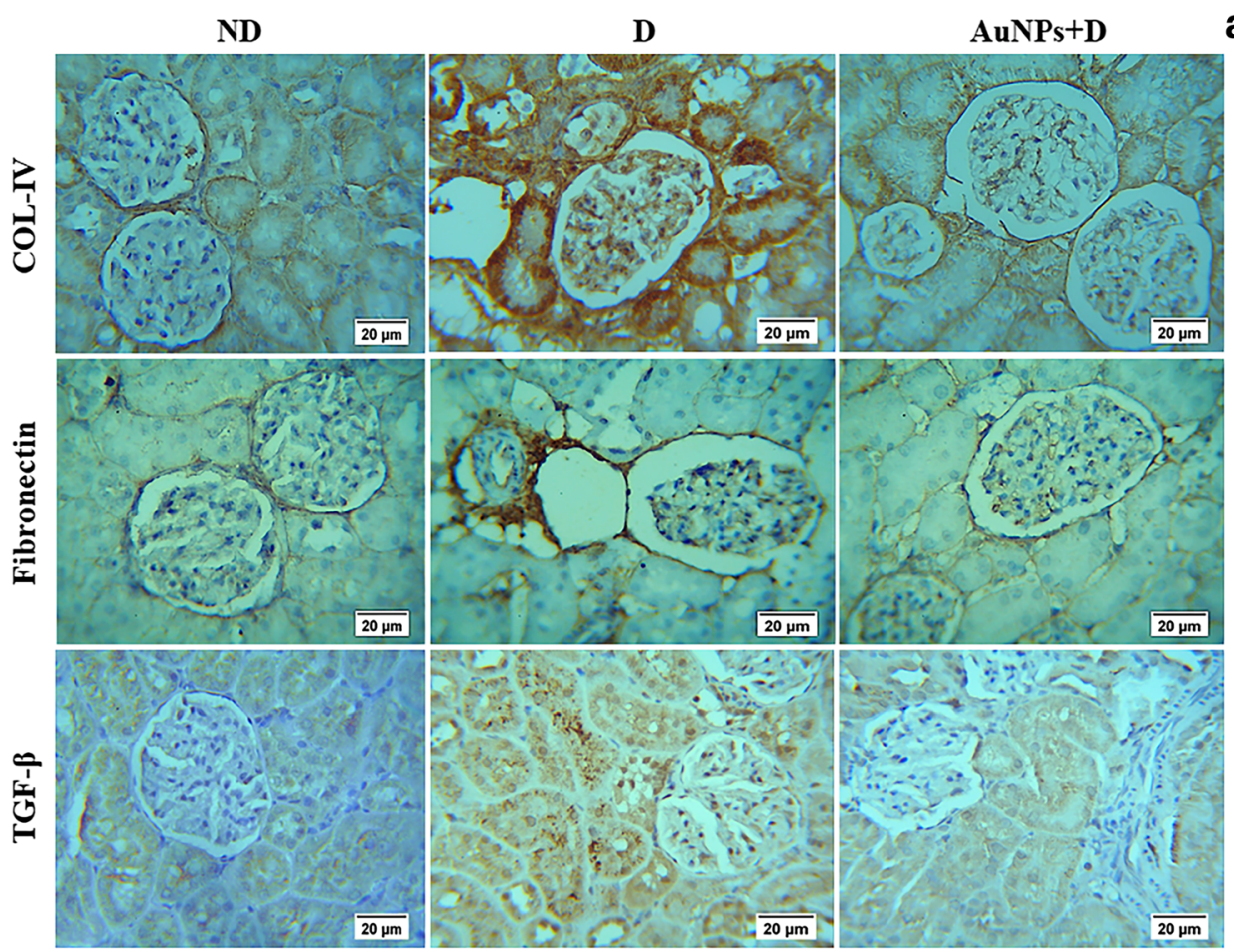

a

b
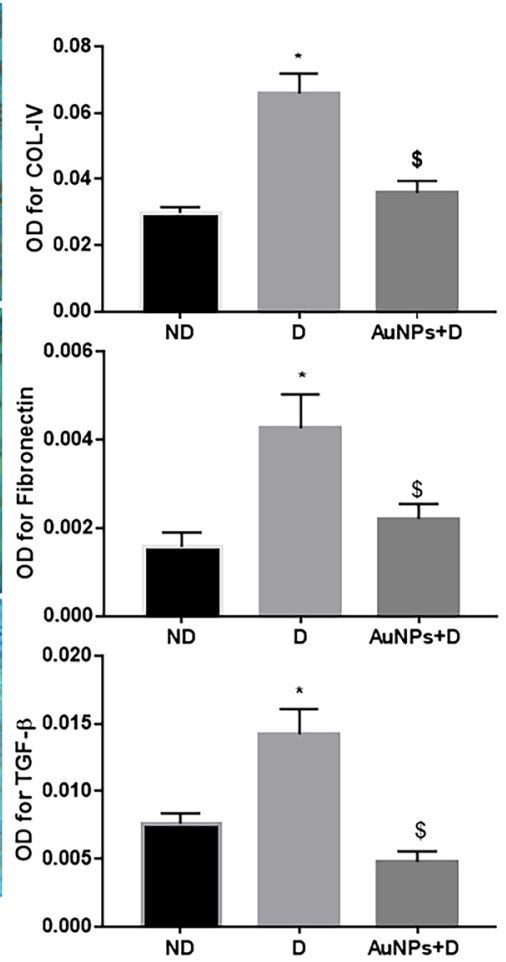

Fig. 3 Immunohistochemistry detects that AuNP treatment inhibits the collagen IV, fibronectin, and TGF- $\beta_{1}$ expression in the diabetic kidney. a Immunohistochemical stain of the kidney sections (magnification, $\times 400$ ) shows that the immunostaining (brown staining) in the glomeruli was much stronger in the D group compared with the ND group. AuNP treatment inhibited the increase in the immunostaining in the AuNPs + D group. b Immunohistochemistry optical density score. Data represent

process, and thickening of the GBM and glomerulosclerosis [2]. Our results revealed that 50-nm AuNPs effectively ameliorated DN in a rat model of STZ-induced type 1 diabetes.

Podocytes play a major role in maintaining the integrity and permeability of the glomerular filtration barrier [8]. In DN, a reduction in podocyte number, the effacement of podocyte foot processes, and the loss of slit diaphragm proteins such as nephrin and podocin result in a leakage of albumin and proteinuria [29]. The results of the present study showed that the levels of urinary albumin were significantly increased in diabetic rats which were associated with the loss of podocytes, widening and effacement of foot process, and decrease in the staining intensity of nephrin and podocin. Our data indicated that AuNPs significantly reduced albuminuria and the podocyte ultrastructural abnormalities and restored the staining intensity of nephrin and podocin, suggesting a protective effect for AuNPs on the structure of podocytes in the diabetic conditions.

In addition, GBM and TBM thickening along with mesangial expansion seem to be a critical determining factors for the progress of proteinuria and the advance of kidney dysfunction in DN [30]. Both mesangial expansion and GBM and TBM thickening are a consequence of ECM protein accumulation due to their increased production, decreased degradation, or both [2]. Our

the mean \pm SEM. (COL-IV $* P<0.05$ compared with the ND group, ${ }^{\$} P<0.0001$ compared with D. Fibronectin $* P<0.05$ compared with the ND group, ${ }^{\$} P=0.0074$ compared with D. TGF- $\beta_{1} * P<0.05$ compared with the ND group, ${ }^{\$} P<0.0001$ compared with D). ND, non-diabetic; D, diabetic; AuNPs, gold nanoparticles; TGF- $\beta_{1}$, transforming growth factor- $\beta_{1}$

results showed that the GBM thickening was significantly lower in the AuNPs $+\mathrm{D}$ rats compared with the D group. In addition, the upregulation of tissue collagen and fibronectin, the major constituent of ECM proteins, in the diabetic kidney is effectively reversed after the AuNP treatment. Many studies support a role for hyperglycemia in the increased synthesis of many ECM proteins that accumulate within the mesangium and GBM of diabetic kidneys resulting in changes in permeability of the filtration barrier [31]. Improved glycemic control in patients with diabetes is, therefore, a goal in the attempt to slow the rate of progression of diabetic renal disease [32]. ECM accumulation in diabetes is induced by TGF- $\beta_{1}$, which enhances collagen, fibronectin, and laminin synthesis and inhibits proteins that mediate the ECM protein degradation [33]. Studies have shown that high glucose environment could increase TGF- $\beta_{1}$ expression in renal tubular epithelial cells and activate ECM protein deposition in glomeruli [34]. Therefore, the TGF- $\beta_{1}$ pathway is a therapeutic target for DN [35]. In this study, TGF- $\beta_{1}$ mRNA and protein levels were increased in the D group and reduced in the AuNP-treated rats, which is in turn reduce the accumulation of the ECM proteins, collagen IV, and fibronectin. This may provide insight into the mechanism whereby AuNP contributes to reduced mesangial matrix expansion in the diabetic condition. Consistent with our 

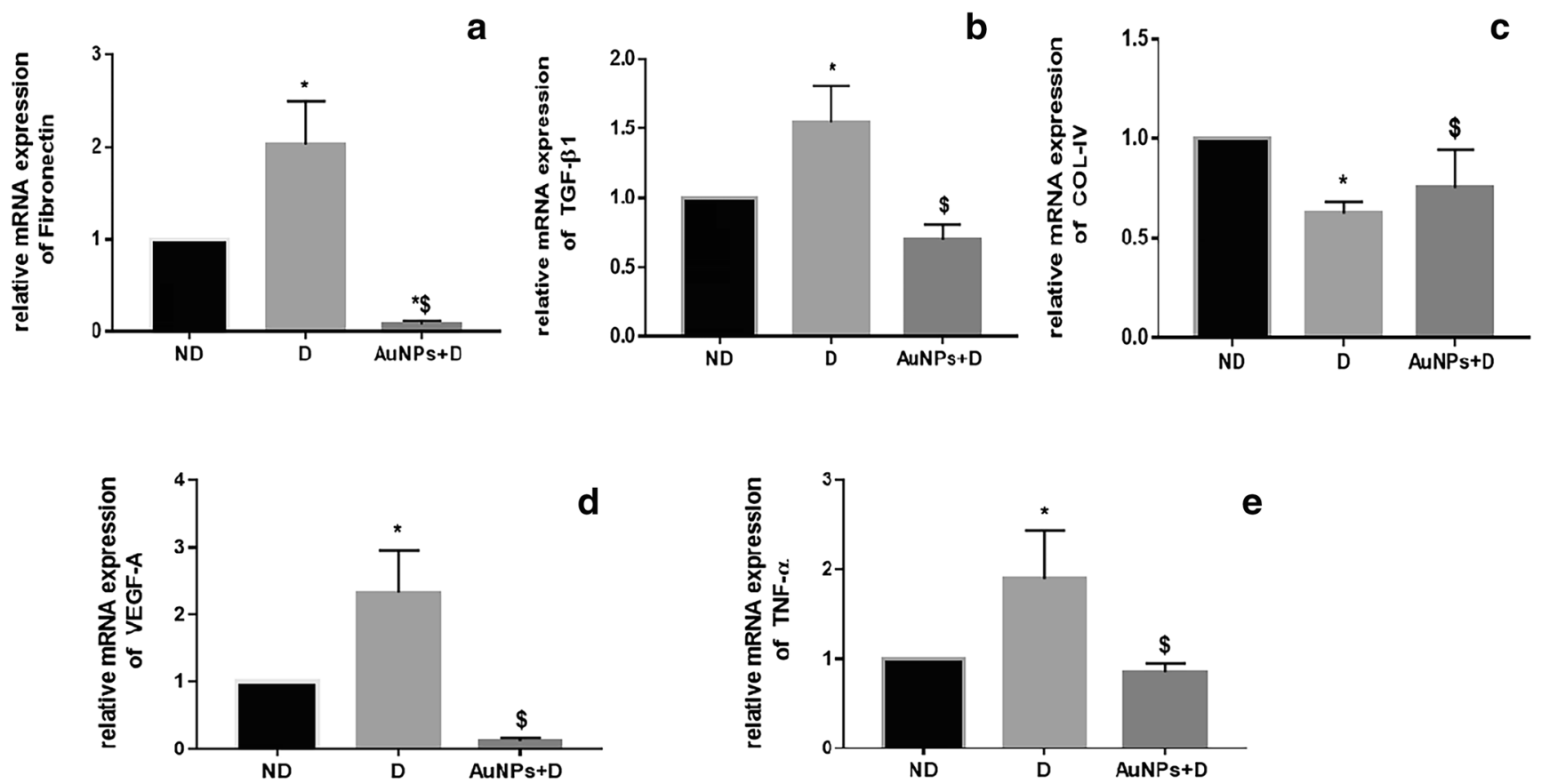

Fig. 4 Effect of AuNP treatment on the mRNA expression in the normal and diabetic kidney. a Fibronectin, $* P<0.05$ compared with the ND group; ${ }^{\$} P<0.0001$ compared with D. b TGF- $\beta_{1}, * P<0.05$ compared with the ND group; ${ }^{\$} P=0.0008$ compared with D. c Collagen IV, $* P<0.05$ compared with the ND group; ${ }^{\$} P=0.15$ compared with D. d VEGF-A, $* P<0.05$ compared with the ND group; ${ }^{\$} P=0.0006$ compared

with D. e TNF- $\alpha ; * P<0.05$ compared with the ND group; ${ }^{\$} P=0.0082$ compared to D. Data represent the mean \pm SEM. ND, non-diabetic; D, diabetic; AuNPs, gold nanoparticles; TGF- $\beta_{1}$, transforming growth factor- $\beta_{1}$; VEGF-A, vascular endothelial growth factor-A; TNF- $\alpha$, tumor necrosis factor- $\alpha$

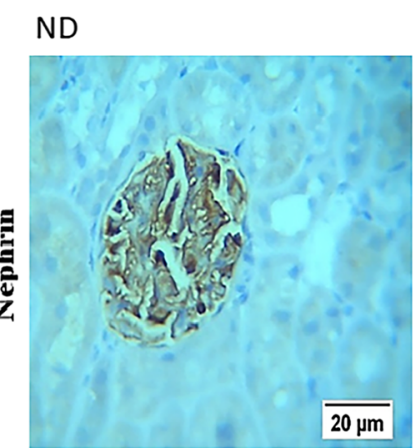

$\mathrm{D}$
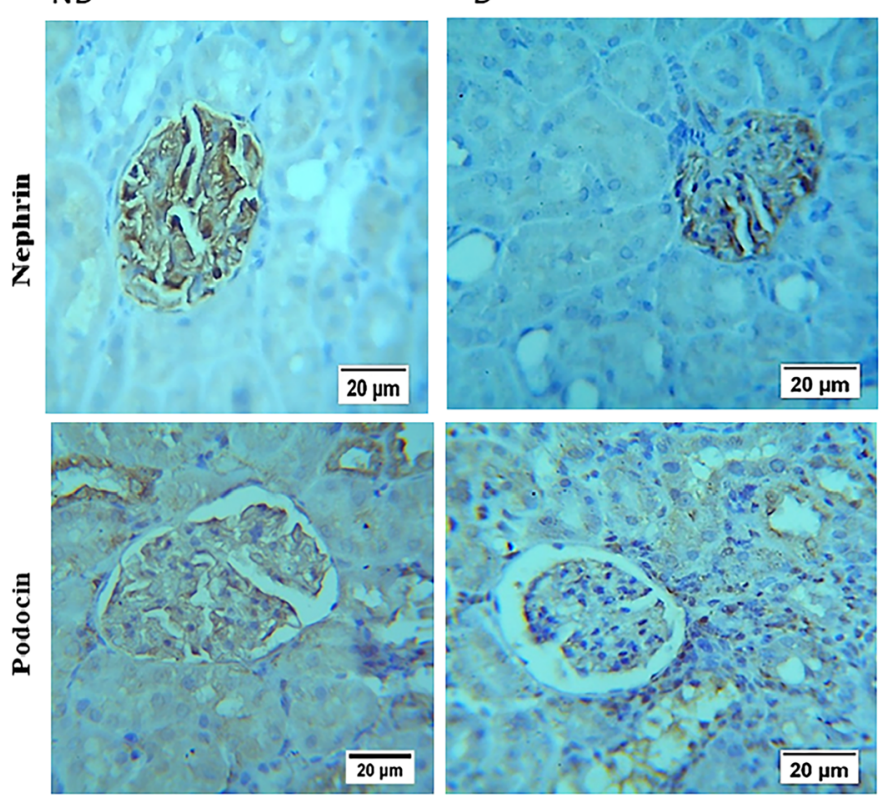

Fig. 5 Immunohistochemistry detects that AuNP treatment restores the nephrin and podocin expression in the diabetic kidney. a Immunohistochemical stain of the kidney sections (magnification, $\times$ 400) shows that the immunostaining (brown staining) in the glomeruli was much stronger in the ND group compared with the D group. AuNP treatment inhibited the decrease in the nephrin and podocin
AuNPs+D
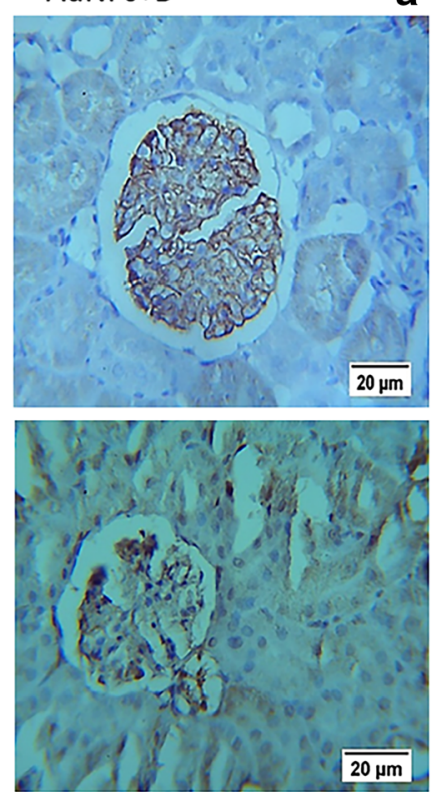

a
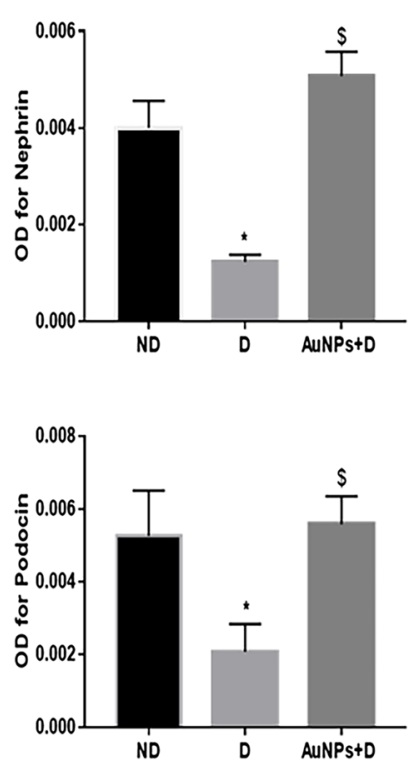

immunostaining in the AuNPs + D group. b Immunohistochemistry optical density score. Data represent the mean \pm SEM. (Nephrin; $* P<0.05$ compared with the ND group. ${ }^{\$} P<0.0001$ compared with D. Podocin; $* P<0.05$ compared with the ND group. ${ }^{\$} P=0.0006$ compared with D). ND, non-diabetic; D, diabetic; AuNPs, gold nanoparticles 
results, AuNPs significantly decreased the expression of fibronectin and TGF- $\beta_{1}$ and inhibit matrix deposition in pancreatic ductal adenocarcinoma [36]. The exogenous administration of AuNPs is documented to afford protection against liver fibrosis [37]. In addition, gold nanorods modulate cell-mediated matrix remodeling and decrease the mRNA expression of type I collagen in neonatal rat cardiac fibroblasts [38].

Oxidative stress, defined as an imbalance in the oxidant/ antioxidant production, has been considered to be one of the primary factors for the development of DN $[15,16]$. Excessive production of reactive oxygen species (ROS) in multiple cell types including mesangial cells and podocytes activates various protein kinases, cytokines, and transcription factors which eventually cause increased expression of ECM proteins and progression to renal fibrosis $[31,39]$. Thus, neutralizing the ROS induced by hyperglycemia is effective in preventing experimentally induced diabetes and its complications such as DN [40]. SOD and catalase are considered the key antioxidant enzymes which detoxify ROS to water in the kidney [1]. In our study, AuNP treatment significantly reduced the renal oxidative stress by increasing the activities of renal SOD and catalase and by reducing the tissue MDA level. These results were in agreement with previous in vivo studies that showed that AuNPs have antioxidative and anti-hyperglycemic activities in diabetic animal models [13, 41].

Several studies have shown the crucial role of kidney inflammation in promoting the development and progression of DN [42-44]. In particular, inflammatory cytokines have emerged as being closely involved in the pathogenesis of DN [45]. For instance, TNF- $\alpha$ has been reported as one of the major inflammatory cytokines that is believed to play an important role in the pathogenesis of DN. TNF- $\alpha$ has been reported to be responsible for the production of ROS, induction of cell injury, and increases in endothelial permeability $[44,45]$. In the present study, AuNPs were found to be associated with the suppression of the inflammation in the AuNPs + D group. Specifically, treatment with AuNPs showed a significant reduction in the mRNA expression levels of TNF- $\alpha$ down to the normal levels as compared with the $\mathrm{D}$ group, indicating the effect of AuNPs on suppressing the inflammation through preventing TNF- $\alpha$ over expression. This result is consistent with other studies which described AuNP as an anti-inflammatory agent in different diseases including diabetes [14, 46-48].

Moreover, vascular endothelial growth factor (VEGF) is the most potent angiogenic factor and its upregulation often is observed in many pathologic conditions including DN [49]. Upregulated VEGF synthesis is accompanied by the glomerular hypertrophy and urinary albumin excretion in the DN $[10,49$, 50]. Several studies have indicated that VEGF mRNA, protein expression, and activity are enhanced in kidneys with early DN [51-54]. In the current study, the level of VEGF-A was significantly increased in the kidney of diabetic rats. However, AuNP treatment successfully reversed the increase in the expression of the VEGF in the AuNPs + D group. Our findings underscored the role of AuNPs as anti-angiogenic treatment, which is consistent with previous findings [55]. Nevertheless, the mechanism of action of the AuNPs as anti-angiogenic factor is not fully understood; however, previous report proposed that the AuNPs inhibit the activity of heparin-binding growth factors (HB-GFs) such as vascular endothelial growth factor 165 (VEGF165) and basic fibroblast growth factor (bFGF) by the change in HB-GF conformation/configuration (denaturation) by the AuNPs [55].

Finally, due to the variability of parameters such as the size and surface charge of the particle, cell type, and dosing parameters, no straightforward deduction has arisen from the available studies regarding AuNP toxicity. However, a great advantage of AuNPs over other nanoparticles is that they show no cytotoxicity in human cells [56]. In the current study, daily administration of 50-nm AuNPs for 7 weeks was well tolerated by the animals (no significant changes in the feed intake, mortality rate, and behavior). In addition, the analysis of hepatic function showed no significant effects of 50-nm AuNP treatment on the serum aspartate aminotransferase (AST) and alanine aminotransferase (ALT) levels (data not shown).

\section{Conclusion}

The results of this study demonstrated a potential curing efficiency of AuNPs on DN. The study outcomes confirmed that STZ treatment clearly raised the blood glucose level which enhanced oxidative stress and inflammation. The adverse effects of these outcomes were evident in renal tissue indicated by the accumulation of ECM proteins, GBM thickening, and podocyte injury which eventually lead to albuminuria. In contrast, AuNPs were able to show promising effects in preventing the adverse effects of hyperglycemia in renal tissue by preventing the accumulation of ECM proteins and the amelioration of podocyte injury through the downregulation of growth factors, inflammatory, and angiogenesis markers. In summary, the current study proposed a potential use of AuNPs for the treatment and prevention of diabetic nephropathy complications; however, future functional studies are required to understand the exact molecular mechanism of action at cellular and physiological levels.

Acknowledgments We sincerely thank the Universiti Teknologi Malaysia for facilitating the use of the transmission electron microscope unit. We also thank Saja Haifawi for her help with the RNA extraction and quantitative real time-PCR experiment. The authors acknowledge Dr. Hamid A. Bakshi from the SAAD Center for Pharmacy and Diabetes, Ulster University, UK for his help in improving images quality.

Funding information This work was supported by the Deanship of Research and Graduate Studies at Yarmouk University (grant number 34/2017). 


\section{Compliance with ethical standards}

Conflict of interest The authors declare that they have no conflicts of interest.

Open Access This article is distributed under the terms of the Creative Commons Attribution 4.0 International License (http:// creativecommons.org/licenses/by/4.0/), which permits unrestricted use, distribution, and reproduction in any medium, provided you give appropriate credit to the original author(s) and the source, provide a link to the Creative Commons license, and indicate if changes were made.

\section{References}

1. Miranda-Díaz AG, Pazarín-Villaseñor L, Yanowsky-Escatell FG, Andrade-Sierra J. Oxidative stress in diabetic nephropathy with early chronic kidney disease. J Diabetes Res. 2016;2016:1-7.

2. Fioretto P, Mauer M, editors. Histopathology of diabetic nephropathy. Seminars in nephrology: Elsevier; 2007.

3. Mooyaart AL. Diabetic nephropathy: pathology, genetics and carnosine metabolism: Department Pathology, Faculty of Medicine/Leiden University Medical Center (LUMC), Leiden University; 2011.

4. Wahba NS, Shaban SF, Kattaia AA, Kandeel SA. Efficacy of zinc oxide nanoparticles in attenuating pancreatic damage in a rat model of streptozotocin-induced diabetes. Ultrastruct Pathol. 2016;40(6): 358-73.

5. Pourghasem M, Shafi H, Babazadeh Z. Histological changes of kidney in diabetic nephropathy. Caspian J Intern Med. 2015;6(3): 120-7.

6. Hu C, Sun L, Xiao L, Han Y, Fu X, Xiong X, et al. Insights into the mechanisms involved in the expression and regulation of extracellular matrix proteins in diabetic nephropathy. Curr Med Chem. 2015;22(24):2858-70.

7. Kitada M, Zhang Z, Mima A, King GL. Molecular mechanisms of diabetic vascular complications. J Diabetes Investig. 2010;1(3):7789.

8. Shankland S. The podocyte's response to injury: role in proteinuria and glomerulosclerosis. Kidney Int. 2006;69(12):2131-47.

9. Ghayur A, Margetts PJ. Transforming growth factor-beta and the glomerular filtration barrier. Kidney Res Clin Pract. 2013;32(1):310 .

10. Zent R, Pozzi A, editors. Angiogenesis in diabetic nephropathy. Seminars in nephrology: Elsevier; 2007.

11. Jo DH, Kim JH, Lee TG, Kim JH. Size, surface charge, and shape determine therapeutic effects of nanoparticles on brain and retinal diseases. Nanomed Nanotechnol. 2015;11(7):1603-11.

12. Pietroiusti A, Campagnolo L, Fadeel B. Interactions of engineered nanoparticles with organs protected by internal biological barriers. Small. 2013;9(9-10):1557-72.

13. Barathmanikanth S, Kalishwaralal K, Sriram M, Pandian SR, Youn HS, Eom S, et al. Anti-oxidant effect of gold nanoparticles restrains hyperglycemic conditions in diabetic mice. J Nanobiotechnol. 2010;8(1):16.

14. Karthick V, Kumar VG, Dhas TS, Singaravelu G, Sadiq AM, Govindaraju K. Effect of biologically synthesized gold nanoparticles on alloxan-induced diabetic rats - an in vivo approach. Colloid Surf B. 2014;122:505-11.

15. King GL. The role of inflammatory cytokines in diabetes and its complications. J Periodontol. 2008;79(8S):1527-34.
16. Rahimi R, Nikfar S, Larijani B, Abdollahi M. A review on the role of antioxidants in the management of diabetes and its complications. Biomed Pharmacother. 2005;59(7):365-73.

17. Manna K, Mishra S, Saha M, Mahapatra S, Saha C, Yenge G, et al. Amelioration of diabetic nephropathy using pomegranate peel extract-stabilized gold nanoparticles: assessment of nf- $\mathrm{kb}$ and $\mathrm{nrf} 2$ signaling system. Int J Nanomedicine. 2019;14:1753-77.

18. Hamza A, Bashuaib H. Anti-diabetic and anti-fibrotic effects of gold and silver nano-particles on diabetic nephropathy induced experimentally. Biosci Res. 2018;15(1):215-28.

19. Choi CH, Zuckerman JE, Webster P, Davis ME. Targeting kidney mesangium by nanoparticles of defined size. Proc Natl Acad Sci U S A. 2011;108(16):6656-61.

20. Al-Trad B, Aljabali A, Al Zoubi M, Shehab M, Omari S. Effect of gold nanoparticles treatment on the testosterone-induced benign prostatic hyperplasia in rats. Int J Nanomedicine. 2019;14:3145-54.

21. Buege JA, Aust SD. [30] microsomal lipid peroxidation. Methods in enzymology: Elsevier; 1978. p. 302-10.

22. Guo M, Ricardo SD, Deane JA, Shi M, Cullen-McEwen L, Bertram JF. A stereological study of the renal glomerular vasculature in the $\mathrm{db} / \mathrm{db}$ mouse model of diabetic nephropathy. J Anat. 2005;207(6): 813-21.

23. van den Bergh Weerman MA, Assmann KJ, Weening JJ, Florquin S. Podocyte foot process effacement is not correlated with the level of proteinuria in human glomerulopathies. Kidney Int. 2004;66(5): 1901-6.

24. Livak KJ, Schmittgen TD. Analysis of relative gene expression data using real-time quantitative per and the $2^{-\Delta \Delta \mathrm{CT}}$ method. Methods. 2001;25(4):402-8.

25. Klemis V, Ghura H, Federico G, Würfel C, Bentmann A, Gretz N, et al. Circulating fibronectin contributes to mesangial expansion in a murine model of type 1 diabetes. Kidney Int. 2017;91(6):1374 85.

26. Daisy P, Saipriya K. Biochemical analysis of cassia fistula aqueous extract and phytochemically synthesized gold nanoparticles as hypoglycemic treatment for diabetes mellitus. Int J Nanomedicine. 2012;7:1189.

27. Dhas TS, Kumar VG, Karthick V, Vasanth K, Singaravelu G, Govindaraju K. Effect of biosynthesized gold nanoparticles by Sargassum swartzii in alloxan induced diabetic rats. Enzym Microb Technol. 2016;95:100-6.

28. Edrees HM, Elbehiry A, Elmosaad YM. Hypoglycemic and antiinflammatory effect of gold nanoparticles in streptozotocin-induced type 1 diabetes in experimental rats. Int J Diabetes Res. 2017;6(1): $16-23$.

29. Arif E, Nihalani D. Glomerular filtration barrier assembly: an insight. Postdoc J. 2013;1(4):33-45.

30. Marshall CB. Re-thinking glomerular basement membrane thickening in diabetic nephropathy-adaptive or pathogenic? Am J Physiol Renal Physiol. 2016.

31. Kanwar YS, Wada J, Sun L, Xie P, Wallner EI, Chen S, et al. Diabetic nephropathy: mechanisms of renal disease progression. Exp Biol Med. 2008;233(1):4-11.

32. Abrass C. Diabetic nephropathy. Mechanisms of mesangial matrix expansion. West J Med. 1995;162(4):318.

33. Loeffler I, Wolf G. Transforming growth factor- $\beta$ and the progression of renal disease. Nephrol Dial Transplant. 2013;29(suppl_1): i37-45.

34. Wang Y-Y, Tang L-Q, Wei W. Berberine attenuates podocytes injury caused by exosomes derived from high glucose-induced mesangial cells through tgf $\beta 1$-pi3k/akt pathway. Eur J Pharmacol. 2018;824:185-92.

35. Scindia YM, Deshmukh US, Bagavant H. Mesangial pathology in glomerular disease: targets for therapeutic intervention. Adv Drug Deliv Rev. 2010;62(14):1337-43. 
36. Saha S, Xiong X, Chakraborty PK, Shameer K, Arvizo RR, Kudgus RA, et al. Gold nanoparticle reprograms pancreatic tumor microenvironment and inhibits tumor growth. ACS Nano. 2016;10(12): 10636-51.

37. de Carvalho TG, Garcia VB, de Araújo AA, da Silva Gasparotto LH, Silva H, Guerra GCB, et al. Spherical neutral gold nanoparticles improve anti-inflammatory response, oxidative stress and fibrosis in alcohol-methamphetamine-induced liver injury in rats. Int J Pharm. 2018;548(1):1-14.

38. Sisco PN, Wilson CG, Mironova E, Baxter SC, Murphy CJ, Goldsmith EC. The effect of gold nanorods on cell-mediated collagen remodeling. Nano Lett. 2008;8(10):3409-12.

39. Kashihara N, Haruna Y, Kondeti VK, Kanwar YS. Oxidative stress in diabetic nephropathy. Curr Med Chem. 2010;17(34):4256-69.

40. Kumar GS, Kulkarni A, Khurana A, Kaur J, Tikoo K. Selenium nanoparticles involve hsp-70 and sirt1 in preventing the progression of type 1 diabetic nephropathy. Chem Biol Interact. 2014;223:12533.

41. Opris R, Tatomir C, Olteanu D, Moldovan R, Moldovan B, David $\mathrm{L}$, et al. The effect of Sambucus nigra L. extract and phytosinthesized gold nanoparticles on diabetic rats. Colloid Surf B. 2017;150:192-200.

42. Duran-Salgado MB, Rubio-Guerra AF. Diabetic nephropathy and inflammation. World J Diabetes. 2014;5(3):393.

43. Lenz O, Fornoni A, Ijaz A, Tejada T. Role of inflammation in diabetic nephropathy. Curr Diabetes Rev. 2008;4(1):10-7.

44. Lim AK, Tesch GH. Inflammation in diabetic nephropathy. Mediat Inflamm. 2012;2012:146154.

45. Donate-Correa J, Martín-Núñez E, Muros-de-Fuentes M, MoraFernández C, Navarro-González JF. Inflammatory cytokines in diabetic nephropathy. J Diabetes Res. 2015;2015:948417.

46. Chen H, Dorrigan A, Saad S, Hare DJ, Cortie MB, Valenzuela SM. In vivo study of spherical gold nanoparticles: inflammatory effects and distribution in mice. PLoS One. 2013;8(2).

47. Paula M, Petronilho F, Vuolo F, Ferreira GK, De Costa L, Santos GP, et al. Gold nanoparticles and/or n-acetylcysteine mediate carrageenan-induced inflammation and oxidative stress in a concentration-dependent manner. J Biomed Mater Res A. 2015;103(10):3323-30.

48. Muller AP, Ferreira GK, Pires AJ, de Bem SG, de Souza DL, de Abreu BJ, et al. Gold nanoparticles prevent cognitive deficits, oxidative stress and inflammation in a rat model of sporadic dementia of Alzheimer's type. Mater Sci Eng C Mater Biol Appl. 2017;77: 476-83.

49. Dronavalli S, Duka I, Bakris GL. The pathogenesis of diabetic nephropathy. Nat Rev Endocrinol. 2008;4(8):444-52.

50. Nakagawa T, Kosugi T, Haneda M, Rivard CJ, Long DA. Abnormal angiogenesis in diabetic nephropathy. Diabetes. 2009;58(7):1471-8.

51. Hohenstein B, Hausknecht B, Boehmer K, Riess R, Brekken R, Hugo C. Local VEGF activity but not VEGF expression is tightly regulated during diabetic nephropathy in man. Kidney Int. 2006;69(9):1654-61.

52. Kim NH, Oh JH, Seo JA, Lee KW, Kim SG, Choi KM, et al. Vascular endothelial growth factor (VEGF) and soluble VEGF receptor flt-1 in diabetic nephropathy. Kidney Int. 2005;67(1):16777.

53. Wolf G, Chen S, Ziyadeh FN. From the periphery of the glomerular capillary wall toward the center of disease: podocyte injury comes of age in diabetic nephropathy. Diabetes. 2005;54(6):1626-34.

54. Al-Trad B, Ashankyty IM, Alaraj M. Progesterone ameliorates diabetic nephropathy in streptozotocin-induced diabetic rats. Diabetol Metab Syndr. 2015;7(1):97.

55. Arvizo RR, Rana S, Miranda OR, Bhattacharya R, Rotello VM, Mukherjee P. Mechanism of anti-angiogenic property of gold nanoparticles: role of nanoparticle size and surface charge. Nanomedicine. 2011;7(5):580-7.

56. Connor EE, Mwamuka J, Gole A, Murphy CJ, Wyatt MD. Gold nanoparticles are taken up by human cells but do not cause acute cytotoxicity. Small. 2005;1(3):325-7.

Publisher's note Springer Nature remains neutral with regard to jurisdictional claims in published maps and institutional affiliations. 